\title{
What is appropriate to post on social media? Ratings from students, faculty members and the public
}

\author{
Anuja Jain, ${ }^{1}$ Elizabeth M Petty, ${ }^{2}$ Reda M Jaber, ${ }^{3}$ Sean Tackett, ${ }^{4}$ Joel Purkiss, ${ }^{3}$ James Fitzgerald ${ }^{5}$
} \& Casey White ${ }^{6}$

\begin{abstract}
OBJECTIVES The purpose of this study was to ascertain what medical students, doctors and the public felt was unprofessional for medical students, as future doctors, to post on a social media site, Facebook ${ }^{\circledR}$. The significance of this is that unprofessional content reflects poorly on a student, which in turn can significantly affect a patient's confidence in that student's clinical abilities.
\end{abstract}

METHODS An online survey was designed to investigate the perceptions of University of Michigan medical students, attending physicians and non-health care university-wide employees (that serves as a subset of the public) regarding mock medical students' Facebook ${ }^{\circledR}$ profile screenshots. For each screenshot, respondents used a 5-point Likert scale to rate 'appropriateness' and whether they would be 'comfortable' having students posting such content as their future doctors.

RESULTS Compared with medical students, faculty members and public groups rated images as significantly less appropriate $(p<0.001)$ and indicated that they would be less comfortable $(\mathrm{p}<0.001)$ having posting students as future doctors. All three groups rated screenshots containing derogatory or private information about patients, followed by images suggesting marijuana use, as least appropriate. Images conveying intimate heterosexual couples were rated as most appropriate. Overall, the doctor group, females and older individuals were less permissive when compared with employee and student groups, males and younger individuals, respectively.

CONCLUSIONS The most significant conclusion of our study is that faculty members, medical students and the 'public' have different thresholds of what is acceptable on a social networking site. Our findings will prove useful for students to consider the perspectives of patients and faculty members when considering what type of content to post on their social media sites. In this way, we hope that our findings provide insight for discussions, awareness and the development of guidelines related to online professionalism for medical students.

Medical Education 2014: 48: 157-169

doi:10.1111/medu.12282

Discuss ideas arising from the article at

'www.mededuc.com discuss'

${ }^{1}$ Children's Hospital Boston/Boston Medical Center, Boston,

Massachusetts, USA

${ }^{2}$ School of Medicine and Public Health, University of Wisconsin-

Madison, Madison, Wisconsin, USA

${ }^{3}$ University of Michigan Medical School, Ann Arbor, Michigan, USA

${ }^{4}$ Johns Hopkins University Bayview Medical Center, Baltimore,

Maryland, USA

${ }^{5}$ Department of Medical Education, University of Michigan

Medical School, Ann Arbor, Michigan, USA

${ }^{6}$ University of Virginia School of Medicine, Charlottesville, Virginia, USA

Correspondence: Casey White, University of Virginia School of Medicine, 200 Jeanette Lancaster Way, PO Box 800380 ,

Charlottesville, Virginia 22908-0380, USA. Tel: 001434924 1681; E-mail: caseywhite@virginia.edu 


\section{INTRODUCTION}

Social media websites, which facilitate exchange of user-generated content, have had a profound impact on the lives of many in a very short time, especially among young adults who find it hard to imagine that Facebook ${ }^{\circledR}$ did not exist before 2004. Today, Facebook ${ }^{\circledR}$ is the most popular website on the Internet ${ }^{1}$ and is now approaching 1 billion users worldwide. ${ }^{2}$ The increased availability and speed of the Internet has made social media present and available virtually all the time. Indeed, almost half a billion people access Facebook ${ }^{\circledR}$ from mobile devices. $^{2}$

\section{Professionalism guidelines on social media}

The medical profession uses social media as much as the general population. ${ }^{3}$ For example, nearly $90 \%$ of practising doctors have Facebook ${ }^{\circledR}$ pages for personal or professional use, ${ }^{4}$ and nearly all medical schools have some Facebook ${ }^{\circledR}$ presence. ${ }^{5}$ Although there are potential health care benefits to social media, such as facilitating online patient communities, professional communities and interactions between patients and their providers, most of the discourse about social media in health care to date has centred on the impact of social media on professionalism and the doctor-patient relationship. ${ }^{6,7}$ The American Medical Association in November 2010 and the American College of Physicians in January $2012^{8}$ published guidelines for maintaining professionalism online. Both advocate extending standards of professionalism to the online environment to maintain appropriate professional boundaries and confidentiality with patient information. ${ }^{8}$ The American Medical Association's policy also recommends online self-monitoring and emphasises keeping personal and professional content separate. ${ }^{8}$ In addition, policies regarding social media sites also exist in international settings. For example, the Australian Medical Association has created a 'Code of Conduct' to help medical students navigate using social media sites in various situations ranging from contact with a patient to defaming another colleague. ${ }^{9}$ Similarly, the General Medical Council in the UK and the Canadian Medical Association have guidelines for medical students regarding social media use. ${ }^{10}$

However, many of these guidelines have been created by practising doctors, and sometimes without the input of medical students. To our knowledge, no guidelines exist that incorporate the views of practising doctors, medical students and the public three important groups of stakeholders. Such guidelines would be difficult to create without consensus regarding the perceptions of what constitutes unprofessional online behaviour among these three groups. In fact, a recent survey of internal medicine educators revealed a broad constellation of behaviours that might be considered inappropriate if depicted online. ${ }^{11}$

\section{Medical students' use of social media}

A 2010 qualitative study found that, with the exception of HIPAA violations and illegal behaviours, there was no consensus among medical students for what constitutes unprofessional behaviour. ${ }^{12}$ Students in that study reported that their postings were generally related to personal matters and personal experiences; one student felt that posting inappropriate material on personal social media sites was 'unavoidable'. In fact, some medical students have reported a sense of being 'watched' by their medical schools and they resent what they perceive to be an intrusion. ${ }^{13}$ Indeed, Thompson and colleagues ${ }^{14}$ found that $70 \%$ of medical students' profiles had photographs with alcohol, with a frequency of $10-50 \%$ of photographs that implied excess drinking. Furthermore, 3 of 10 profiles of medical students had 'unprofessional' content readily available to view, such as drunkenness, overt sexuality, foul language and patient privacy violations in non-US locations. In a national survey of allopathic medical schools, $60 \%$ of them reported having had to address students who posted unprofessional content (including breaches of patient confidentiality) online. ${ }^{15}$ Garner and O'Sullivan ${ }^{16}$ surveyed medical students, $52 \%$ of whom admitted to posting photographs on Facebook ${ }^{\circledR}$ they considered embarrassing. ${ }^{16}$ However, students have reported that they do not want or need formal policies for posting content online,${ }^{12}$ in spite of the fact that consequences from violations of e-professionalism in medicine have escalated from academic restrictions to loss of licensure. ${ }^{7}$

\section{Professional identity formation}

Medical students, particularly early on, do not view professionalism in the same way that doctors do, because they are at a very different stage of development. Jarvis-Selinger and colleagues, ${ }^{17}$ using developmental theory as a foundation for describing identity formation, reported that medical trainees do not have a deep understanding of what it actually means to be a doctor; theirs is a "narrowly 
defined, superficial understanding'. ${ }^{17}$ Framed within the context of e-professionalism as an essential element of medical students' identity formation, Kaczmarcyzk and colleagues ${ }^{18}$ cautioned that, due to growing up sharing all types of information, personal and otherwise, using electronic media, it may be difficult for these 'digital natives' to develop boundaries that separate their personal lives from their professional lives.

\section{Social media surveys conducted}

Studies involving focus groups, surveys and reviews of the literature have gathered usage information and perspectives from medical students, residents and doctors. ${ }^{11,12,16,19,20}$ However, to our knowledge, no published study has asked what members of the general public, all of whom are current or potential patients, would consider as appropriate online behaviour for their future doctors. Given that the ultimate goal of maintaining professionalism is to retain excellent patient-doctor relationships, it is imperative to know if current professionalism opinion aligns with the opinions of patients.

This is why we believe our study is important. It is original in that we explored not only perceptions of doctors and medical students, but also among a group of non-health care professional employees that represented, in our opinion, a subset of the public. The aim of the study was to compare these three groups' perceptions of simulated Facebook ${ }^{\circledR}$ postings by medical students. In doing so, we hoped to understand both similarities and differences in opinions of what is considered to be 'unprofessional' Facebook ${ }^{\circledR}$ content by these three groups. Including members of the public in the study is key because there is suspicion and pessimism among social media users as to what is truly private. Most are aware that conversations, photographs and other materials posted to these sites are accessible to a larger and largely unknown audience than ever intended, and the content never really goes away. ${ }^{12,18}$ The findings from this study will be key to medical educators and experts in the field who might use them to create a more meaningful set of guidelines for social media use by medical students.

\section{METHODS}

\section{Data collection}

A survey of currently enrolled medical students, medical school faculty members and non-doctor/non-student employees ('public') from all schools and units of the entire University of Michigan system was conducted in the winter of 2009. Our public population was not limited to the health system or medical school, and respondents in this category who were health care professionals were excluded from the analysis.

The survey was sent by e-mail via dedicated university-based list serves, with two follow-up e-mail reminders. Three different list serves were used: University of Michigan medical students, University of Michigan Medical School faculty members and employees of the University of Michigan. Data collection was carried out using the SurveyGizmo platform, which accumulated all responses as the respondents completed the surveys. Upon completion of the survey, all respondents had the option of entering into a drawing for one of five $\$ 20$ gift certificates and one $\$ 100$ gift card to Amazon.com, funded by the Office of Student Programs at the medical school. To preserve anonymity, Internet Protocol addresses were not used for any other purpose. Responses were confidential and accessible only by the principal investigator using a passwordprotected profile.

\section{Subjects}

In total, 1546 people responded to the survey that was sent out to three list serves. As this was an online survey with list serve e-mail recruitment (i.e. we estimate that thousands could have accessed the survey through the list serve, but we have no way of determining how many deleted it before opening or how many opened and then deleted it), response percentages cannot be accurately determined.

Respondents were first asked to identify themselves as health care professionals or non-health care professionals; health care professionals were asked what type they were (e.g. medical student, medical faculty member, other faculty member, other health care professionals of various types).

We excluded 125 records for respondents who either did not indicate their status or gender or whose self-reported status did not meet our criteria. Therefore, we ultimately included 1421 records in our analysis, representing those respondents classified as medical students $(n=237)$, medical school faculty member $(n=206)$ and the non-health care professional employees or 'public' $(n=978)$. Some respondents provided partial responses to the survey, so the sample size was reduced accordingly for various portions of our analysis (e.g. as eight 
respondents provided either partial or no image rating data, the corresponding analysis had $n=1413$ ).

\section{Survey design}

Several of the study investigators, including medical school students and faculty members, designed the survey instrument based on what has been reported in existing literature and investigator hypotheses. ${ }^{10}$ SurveyGizmo, an online survey program, was used to administer the survey. The survey had four main sections: demographic information, Facebook ${ }^{\circledR}$ usage patterns, attitudes towards online professionalism and a series of 29 fabricated mock Facebook ${ }^{\circledR}$ screenshots, arranged in random order. The mock screenshots were created by using actual Facebook ${ }^{\circledR}$ screenshots as templates. Templates included features that are commonly used to share information on the social networking site, such as a profile picture, an album picture and a status update (Fig. S1). Pictures inserted into the templates were retrieved from websites, such as www.flickr.com, and had the appropriate Creative Commons licenses. We attempted to capture the following major themes with our screenshots picture selection: same-sex pairs (posing together or kissing), opposite-sex pairs (posing together or kissing), comments on patients (e.g. 'saw the fattest patient today'), substance use (students using marijuana, drinking alcohol, smoking cigarettes), comments on medical school (e.g. 'just bombed the anatomy final'), parties or dancing, partial nudity, clinical picture-domestic and clinical picture-international. The clinical picturedomestic category was any screenshot that depicted or described a clinical experience in the USA, such as a picture of a surgical procedure specified to be taken in the USA, whereas clinical picture-international represented an international experience, such as a picture of a student with a patient in hospital in Peru. We intentionally chose pictures of individuals from a wide range of ethno-cultural backgrounds. In addition, we attempted to choose images of individuals engaging in similar behaviours (e.g. kissing) in the same-sex pairs and opposite-sex pairs to avoid confounding variables.

Participants were told that the screenshots were taken from Facebook ${ }^{\circledR}$ profiles of medical students with 'public' privacy settings and thus accessible by search engines and all Facebook ${ }^{\circledR}$ users, and they were told that the screenshots did not display any medical students from the University of Michigan. Participants were asked to review the screenshots, read the text descriptions within these mock posts, as well as the survey question prompts, and then to rate the pictures accordingly. They rated the 'appropriateness' of each screenshot on a Likert scale from 1 (very appropriate to post on an online network) to 5 (not at all appropriate to post on an online network). The subjects were also asked to respond to the question 'Would you feel comfortable having the student who posted this as your doctor in the future?' using a Likert scale ranging from 1 (would be very comfortable having this student as my doctor in the future) to 5 (absolutely would not feel comfortable having this student as my doctor in the future). To facilitate ease of interpretation of study outcomes, these scales were later reversecoded during the analysis stage (so a lower mean score could be interpreted as a lower appropriateness rating or a lower comfort rating). The survey also included questions that elicited open-ended text responses (such as, 'How would you define professionalism?') that are not reported on in this article.

The instrument underwent pilot testing by 20 medical faculty members, 20 medical students from external institutions and 20 non-health professionals (public); these results were not used in the final data analysis. A link to the final survey, including screenshots, can be found here: http://www-personal. umich.edu/ purkissj/FacebookSurvey.pdf.

This study was granted exemption from informed consent by the institutional review board of the University of Michigan Health System. It should be noted that our survey specifically stated that the screenshots did not have pictures of University of Michigan medical students. However, we did not explicitly explain to participants that the screenshots were not real and instead were created by the researchers. This was done so that we could determine the impressions of the participants as if the profile pictures were real.

\section{Statistical analysis}

We calculated descriptive statistics for demographic data (Table 1) and Facebook ${ }^{\circledR}$ usage patterns (Table 2). To determine whether respondent groups differed in their uses of Facebook ${ }^{\circledR}$ we conducted chi-squared tests of significance and tabulated Cramer's $V$ effect size measures. Cramer's $V=\sqrt{ }\left(X^{2} /(n[k-1])\right.$, where $k=$ the smaller of the number of rows or columns. We interpret effect sizes from Cramer's $V$ using the following conventions: $0.1=$ small, $0.3=$ moderate, $0.5=$ large (Table 2). To identify possible group differences in scale-based assessment of the mock Facebook ${ }^{\circledR}$ 
Table 1 Respondent demographic characteristics (unless noted otherwise, results presented as: count, \%)

\begin{tabular}{|c|c|c|c|c|}
\hline & All respondents & Medical students & Medical faculty & Public (non-HCPs) \\
\hline Total & $N=1421$ & $237,16.7$ & $206,14.5$ & $978,68.8$ \\
\hline \multicolumn{5}{|l|}{ Age } \\
\hline mean, (range in years) & $38.8,(19-76)$ & $25.4,(20-38)$ & $45.1,(29-76)$ & 40.8, (19-70) \\
\hline \multicolumn{5}{|l|}{ Sex } \\
\hline Male & $526,37.0$ & $102,43.0$ & $114,55.3$ & $310,31.2$ \\
\hline Female & $895,63.0$ & $135,57.0$ & $92,44.7$ & $668,68.3$ \\
\hline \multicolumn{5}{|l|}{ Race } \\
\hline White & $1110,78.7$ & $154,65.0$ & $165,80.1$ & $791,80.9$ \\
\hline Black & $52,3.7$ & $5,2.1$ & $2,1.0$ & $45,4.6$ \\
\hline Hispanic & $33,2.3$ & $5,2.1$ & $3,1.5$ & $25,2.6$ \\
\hline Asian & $188,13.3$ & $65,27.4$ & $32,15.5$ & $91,9.3$ \\
\hline Mixed* & $28,2.0$ & $5,2.1$ & $3,1.5$ & $20,2.0$ \\
\hline No response & $10,0.7$ & $3,1.3$ & $1,0.5$ & $6,6.1$ \\
\hline \multicolumn{5}{|l|}{ Year in med school } \\
\hline $1^{\text {st }}$ & & $71,30.0$ & & \\
\hline $2^{\text {nd }}$ & & $22,9.3$ & & \\
\hline $3^{\text {rd }}$ & & $64,27.0$ & & \\
\hline $4^{\text {th }}$ & & $73,30.8$ & & \\
\hline Other & & $7,3.0$ & & \\
\hline
\end{tabular}

screenshots, we performed a series of independentsamples Kruskal-Wallis $H^{\prime}$ tests (the $H^{\prime}$ statistic is similar to $H$, but is adjusted for tie rankings). We utilised non-parametric techniques for these data, as some of our scale-based measures showed evidence of non-normal distributions. When statistically significant associations existed, we followed with Dunn-Bonferroni-adjusted post hoc comparisons to determine which groups differed significantly. To measure effect size for these associations, we used eta-squared $\left(\eta^{2}=H^{\prime} /[n-1]\right)$. We interpret effect sizes from eta-squared using the following conventions: $0.01=$ small, $0.06=$ moderate, $0.14=$ large (Table 3). Finally, we conducted two multiple linear least-squares regression analyses to explore the relationship between overall assessments of the screenshots, respondent group and demographic characteristics. We elected to use simultaneous forced entry of all regression variables (i.e. enter method, with all variables included), given the absence of a theoretical framework that might guide a hierarchical approach to adding regression variables. This approach to multivariate regression analyses allowed us to explore the relationship between membership in the respondent groups and appropriateness and comfort ratings, while controlling for additional variables, including respondent gender, race ethnicity and age. To measure effect size for the regression analyses, we used Cohen's $f^{2}\left(f^{2}=\left(R^{2} /\left[1-R^{2}\right]\right)\right.$. We interpret effect sizes from Cohen's $f^{2}$ using the following conventions: $0.02=$ small, $0.15=$ moderate, $0.35=$ large $($ Table 4$)$. Throughout the study, we used $p<0.05$ as our threshold for statistical significance. Statistical analysis was carried out in sPss STATISTICS 17.0 and sPSS/PASW 19 (SPSS, Inc., Chicago, IL, USA).

\section{RESULTS}

\section{Demographics}

Of the 1421 responses analysed, 237 (16.7\%) were medical students, 206 (14.5\%) were medical faculty members and 978 (68.8\%) were members of the public. The mean age of the overall study sample was 38.8 years (range 19-79). Most respondents were White $(78.1 \%)$ and females were overrepresented overall $(63 \%)$. Among medical 
Table 2 Respondent utilisation of Facebook ${ }^{\circledR}$ by group*

\begin{tabular}{|c|c|c|c|c|c|c|c|c|}
\hline & $\begin{array}{l}\text { Total } \\
(n=1421) \%\end{array}$ & $\begin{array}{l}\text { Medical } \\
\text { students } \\
(n=237) \%\end{array}$ & $\begin{array}{l}\text { Medical faculty } \\
\text { members } \\
(n=206) \%\end{array}$ & $\begin{array}{l}\text { Public (non-health } \\
\text { care professionals) } \\
\text { (n=978) } \%\end{array}$ & $\chi^{2}$ & d.f. & p Value & Cramer's V \\
\hline \multicolumn{9}{|c|}{ Networking } \\
\hline Yes & 33.1 & 28.4 & 20.5 & 36.5 & 22.7 & 2 & $<0.001$ & 0.13 \\
\hline No & 66.9 & 71.6 & 79.5 & 63.5 & & & & \\
\hline \multicolumn{9}{|c|}{ Keeping in touch with friends } \\
\hline Yes & 92.1 & 98.2 & 88.6 & 91.0 & 16.8 & 2 & $<0.001$ & 0.11 \\
\hline No & 7.9 & 1.8 & 11.4 & 9.0 & & & & \\
\hline \multicolumn{9}{|c|}{ Communicating with classmates/colleagues } \\
\hline Yes & 48.2 & 64.9 & 30.3 & 46.6 & 54.4 & 2 & $<0.001$ & 0.20 \\
\hline No & 51.8 & 35.1 & 69.7 & 53.4 & & & & \\
\hline \multicolumn{9}{|c|}{ Meeting new people } \\
\hline Yes & 4.6 & 2.7 & 1.5 & 5.7 & 9.8 & 2 & 0.007 & 0.08 \\
\hline No & 95.4 & 97.3 & 98.5 & 94.3 & & & & \\
\hline \multicolumn{9}{|c|}{ Sharing pictures } \\
\hline Yes & 60.2 & 73.0 & 44.7 & 59.1 & 36.7 & 2 & $<0.001$ & 0.16 \\
\hline No & 39.8 & 27.0 & 55.3 & 40.9 & & & & \\
\hline \multicolumn{9}{|l|}{ Fun } \\
\hline Yes & 35.5 & 40.1 & 19.7 & 36.8 & 24.8 & 2 & $<0.001$ & 0.13 \\
\hline No & 64.5 & 59.9 & 80.3 & 63.2 & & & & \\
\hline \multicolumn{9}{|l|}{ Other } \\
\hline Yes & 11.2 & 4.1 & 8.3 & 13.7 & 19.3 & 2 & $<0.001$ & 0.12 \\
\hline No & 88.8 & 95.9 & 91.7 & 86.3 & & & & \\
\hline
\end{tabular}

students, those in the second year of the MD programme were under-represented $(9.3 \%)$ (Table 1$)$.

\section{Facebook $^{\circledR}$ utilisation}

As shown in Table 2, respondent groups reported different types of Facebook ${ }^{\circledR}$ utilisation, with statistically significant group differences for each usage type ( $p<0.05$ in every case) and effect sizes in the weak-to-moderate range (Cramer's $V$ ranged from 0.08 to 0.20 ). Members of the public group were most likely to use Facebook ${ }^{\circledR}$ for networking $\left(36.5 \% ; \chi^{2}=22.7\right.$, d.f. $=2, \mathrm{p}<0.001$, Cramer's $V=0.13$ ), whereas medical students were more likely to use Facebook ${ }^{\circledR}$ for keeping in touch with friends $\left(98.2 \% ; \chi^{2}=16.8\right.$, d.f. $=2, \mathrm{p}<0.001$, Cramer's $V=0.11)$. Use for communicating with classmates/colleagues showed one of the largest discrepancies, with students reporting a $64.9 \%$ rating and faculty members reporting a $30.3 \%$ rating $\left(\chi^{2}=54.4\right.$, d.f. $=2, \mathrm{p}<0.001$, Cramer's $V=0.20)$. Although relatively few respondents, overall, indicated using Facebook ${ }^{\circledR}$ for meeting new people, the public reported the highest rate for this category $\left(5.7 \% ; \chi^{2}=9.8\right.$, d.f. $=2, p=0.007$, Cramer's $V=0.08)$. Medical students indicated most frequently that they used Facebook ${ }^{\circledR}$ to share pictures $\left(73.0 \% ; \chi^{2}=36.7\right.$, d.f. $=2, \mathrm{p}<0.001$, Cramer's $V=0.16)$ and for fun $\left(40.1 \% ; \chi^{2}=24.8\right.$, d.f. $=2, \mathrm{p}<0.001$, Cramer's $V=0.13)$, whereas the public were most likely to use Facebook ${ }^{\circledR}$ for other purposes $\left(13.7 \% ; \chi^{2}=19.3\right.$, d.f. $=2$, $\mathrm{p}<0.001$, Cramer's $V=0.12$ ) (Table 2).

\section{Screenshot ratings}

Data presented in Table 3 show that, overall, faculty members and the public were more conservative 
Table 3 Association between respondent group and evaluation of Facebook ${ }^{\circledR}$ content

\section{Kruskal-Wallis $\mathrm{H}^{\prime}$ and Dunn-Bonferroni Post-Hoc Tests*}

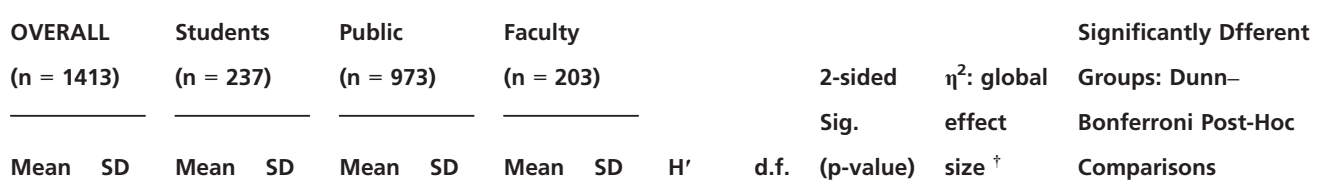

Appropriateness ratings (lower mean scores indicate lower appropriateness ratings) ${ }^{\text {t.s }}$

\begin{tabular}{|c|c|c|c|c|c|c|c|c|c|c|c|c|c|}
\hline \multirow{3}{*}{ OVERALL (29-image average) } & 2.69 & 0.66 & 2.84 & 0.60 & 2.68 & 0.69 & 2.53 & 0.58 & 28.62 & 2 & $<0.001$ & 0.02 & S vs. $P(p=0.005)$ \\
\hline & & & & & & & & & & & & & S vs. $F(p<0.001)$ \\
\hline & & & & & & & & & & & & & P vs. $F(p=0.001)$ \\
\hline \multirow{2}{*}{$\begin{array}{l}\text { Comments on Patients } \\
\text { (4- image average) }\end{array}$} & 1.88 & 0.76 & 1.97 & 0.77 & 1.88 & 0.78 & 1.79 & 0.67 & 5.63 & 2 & 0.060 & $<0.01$ & None - NSD \\
\hline & & & & & & & & & & & & & \\
\hline \multirow[t]{2}{*}{ Marijuana (1 image) } & 1.94 & 1.06 & 2.03 & 1.08 & 1.98 & 1.08 & 1.66 & 0.91 & 18.22 & 2 & $<0.001$ & 0.01 & S vs. $F(p<0.001)$ \\
\hline & & & & & & & & & & & & & P vs. $F(p<0.001)$ \\
\hline \multirow{3}{*}{$\begin{array}{l}\text { Partial Nudity } \\
\text { (7-image average) }\end{array}$} & 2.36 & 0.81 & 2.52 & 0.77 & 2.36 & 0.84 & 2.20 & 0.72 & 16.98 & 2 & $<0.001$ & 0.01 & S vs. $F(p=0.018)$ \\
\hline & & & & & & & & & & & & & S vs. $F(p<0.001)$ \\
\hline & & & & & & & & & & & & & P vs. $F(p=0.036)$ \\
\hline \multirow{2}{*}{$\begin{array}{l}\text { Clinical Picture - Domestic } \\
\text { (2-image average) }\end{array}$} & 2.48 & 1.14 & 2.33 & 1.14 & 2.51 & 1.16 & 2.48 & 1.01 & 5.33 & 2 & 0.070 & $<0.01$ & None - NSD \\
\hline & & & & & & & & & & & & & \\
\hline \multirow{3}{*}{$\begin{array}{l}\text { Same-Sex Pairs } \\
\text { (4-image average) }\end{array}$} & 2.58 & 0.88 & 2.82 & 0.83 & 2.56 & 0.90 & 2.39 & 0.76 & 29.38 & 2 & $<0.001$ & 0.02 & S vs. $P(p<0.001)$ \\
\hline & & & & & & & & & & & & & S vs. $F(p<0.001)$ \\
\hline & & & & & & & & & & & & & P vs. $F(p=0.023)$ \\
\hline \multirow{2}{*}{$\begin{array}{l}\text { Clinical Picture - } \\
\text { International (1 image) }\end{array}$} & 2.59 & 1.42 & 2.08 & 1.19 & 2.80 & 1.44 & 2.15 & 1.30 & 70.73 & 2 & $<0.001$ & 0.05 & S vs. $P(p<0.001)$ \\
\hline & & & & & & & & & & & & & P vs. $F(p<0.001)$ \\
\hline \multirow[t]{3}{*}{ Alcohol (8-image average) } & 2.70 & 0.87 & 2.87 & 0.84 & 2.69 & 0.89 & 2.52 & 0.75 & 22.68 & 2 & $<0.001$ & 0.02 & $S$ vs. $P(p=0.003)$ \\
\hline & & & & & & & & & & & & & S vs. $F(p<0.001)$ \\
\hline & & & & & & & & & & & & & P vs. $F(p=0.014)$ \\
\hline \multirow[t]{2}{*}{ Cigarettes (1 image) } & 2.80 & 1.11 & 2.95 & 1.09 & 2.83 & 1.13 & 2.52 & 1.00 & 16.99 & 2 & $<0.001$ & 0.01 & S vs. $F(p<0.001)$ \\
\hline & & & & & & & & & & & & & P vs. $F(p=0.001)$ \\
\hline \multirow{3}{*}{$\begin{array}{l}\text { Parties or Dancing } \\
\text { (2-image average) }\end{array}$} & 3.35 & 0.94 & 3.60 & 0.86 & 3.33 & 0.96 & 3.16 & 0.86 & 28.21 & 2 & $<0.001$ & 0.02 & S vs. $P(p<0.001)$ \\
\hline & & & & & & & & & & & & & S vs. $F(p<0.001)$ \\
\hline & & & & & & & & & & & & & P vs. $F(p=0.024)$ \\
\hline \multirow{2}{*}{$\begin{array}{l}\text { Comments on Medical } \\
\text { School (4-image average) }\end{array}$} & 3.36 & 0.90 & 3.78 & 0.77 & 3.27 & 0.92 & 3.25 & 0.76 & 67.85 & 2 & $<0.001$ & 0.05 & S vs. $P(p<0.001)$ \\
\hline & & & & & & & & & & & & & S vs. $F(p<0.001)$ \\
\hline \multirow{3}{*}{$\begin{array}{l}\text { Opposite-Sex Pairs } \\
\text { (3-image average) }\end{array}$} & 3.66 & 0.73 & 3.87 & 0.63 & 3.65 & 0.76 & 3.50 & 0.65 & 31.01 & 2 & $<0.001$ & 0.02 & S vs. $P(p<0.001)$ \\
\hline & & & & & & & & & & & & & S vs. $F(p<0.001)$ \\
\hline & & & & & & & & & & & & & P vs. $F(p=0.009)$ \\
\hline OVERALL & 2.75 & 0.68 & 3.03 & 0.63 & 2.70 & 0.70 & 2.67 & 0.54 & 51.44 & 2 & $<0.001$ & 0.04 & S vs. $P(p<0.001)$ \\
\hline (29-image average) & & & & & & & & & & & & & S vs. $F(p<0.001)$ \\
\hline \multirow{3}{*}{$\begin{array}{l}\text { Comments on Patients } \\
\text { (4-image average) }\end{array}$} & 2.04 & 0.80 & 2.34 & 0.84 & 1.96 & 0.80 & 2.07 & 0.64 & 48.49 & 2 & $<0.001$ & 0.03 & S vs. $P(p<0.001)$ \\
\hline & & & & & & & & & & & & & S vs. $F(p=0.022)$ \\
\hline & & & & & & & & & & & & & P vs. $F(p=0.009)$ \\
\hline
\end{tabular}


Table 3 (Continued)

Kruskal-Wallis $\mathrm{H}^{\prime}$ and Dunn-Bonferroni Post-Hoc Tests*

\begin{tabular}{|c|c|c|c|c|c|c|c|c|c|c|c|c|c|}
\hline & & & & & & & & & \multicolumn{5}{|c|}{ Kruskal-Wallis $\mathrm{H}^{\prime}$ and Dunn-Bonferroni Post-Hoc Tests* } \\
\hline & \multicolumn{2}{|c|}{$\begin{array}{l}\text { OVERALL } \\
(n=1413)\end{array}$} & \multicolumn{2}{|c|}{$\begin{array}{l}\text { Students } \\
(\mathrm{n}=237)\end{array}$} & \multicolumn{2}{|c|}{$\begin{array}{l}\text { Public } \\
\text { (n=973) }\end{array}$} & \multicolumn{2}{|c|}{$\begin{array}{l}\text { Faculty } \\
(n=203)\end{array}$} & \multirow[b]{2}{*}{$\mathbf{H}^{\prime}$} & \multirow[b]{2}{*}{ d.f. } & \multirow{2}{*}{$\begin{array}{l}\text { 2-sided } \\
\text { Sig. } \\
\text { (p-value) }\end{array}$} & \multirow{2}{*}{$\begin{array}{l}\eta^{2} \text { : global } \\
\text { effect } \\
\text { size }^{\dagger}\end{array}$} & \multirow{2}{*}{$\begin{array}{l}\text { Significantly Dfferent } \\
\text { Groups: Dunn-- } \\
\text { Bonferroni Post-Hoc } \\
\text { Comparisons }\end{array}$} \\
\hline & Mean & SD & Mean & SD & Mean & SD & Mean & SD & & & & & \\
\hline \multirow[t]{2}{*}{ (1 image average) } & 2.15 & 1.10 & 2.49 & 1.12 & 2.12 & 1.12 & 1.85 & 0.85 & 35.66 & 2 & $<0.001$ & 0.03 & $\begin{array}{l}\text { S vs. } P(p<0.001) \\
\text { S vs. } F(p<0.001)\end{array}$ \\
\hline & & & & & & & & & & & & & P vs. $F(p=0.017)$ \\
\hline Cigarettes (1 image) & 2.57 & 1.08 & 2.74 & 1.08 & 2.56 & 1.11 & 2.42 & 0.92 & 9.08 & 2 & 0.011 & 0.01 & S vs. $F(p=0.010)$ \\
\hline $\begin{array}{l}\text { Partial Nudity } \\
\text { (7-image average) }\end{array}$ & 2.60 & 0.87 & 2.84 & 0.78 & 2.56 & 0.91 & 2.50 & 0.70 & 24.24 & 2 & $<0.001$ & 0.02 & $\begin{array}{l}\text { S vs. } F(p<0.001) \\
\text { S vs. } P(p<0.001)\end{array}$ \\
\hline $\begin{array}{l}\text { Alcohol } \\
\text { (8-image average) }\end{array}$ & 2.74 & 0.86 & 3.03 & 0.81 & 2.69 & 0.89 & 2.64 & 0.67 & 37.02 & 2 & $<0.001$ & 0.03 & $\begin{array}{l}\text { S vs. } F(p<0.001) \\
\text { S vs. } P(p<0.001)\end{array}$ \\
\hline $\begin{array}{l}\text { Same-Sex Pairs } \\
\text { (4-image average) }\end{array}$ & 2.74 & 0.92 & 3.06 & 0.81 & 2.68 & 0.96 & 2.63 & 0.72 & 42.35 & 2 & $<0.001$ & 0.03 & $\begin{array}{l}\text { S vs. } F(p<0.001) \\
\text { S vs. } P(p<0.001)\end{array}$ \\
\hline $\begin{array}{l}\text { Clinical Picture - Domestic } \\
\text { (2-image average) }\end{array}$ & 2.76 & 1.11 & 2.83 & 1.13 & 2.74 & 1.14 & 2.78 & 0.91 & 1.78 & 2 & 0.411 & $<0.01$ & None - NSD \\
\hline $\begin{array}{l}\text { Clinical Picture } \\
\text {-International (1 image) }\end{array}$ & 3.04 & 1.31 & 2.90 & 1.24 & 3.13 & 1.35 & 2.78 & 1.15 & 15.56 & 2 & $<0.001$ & 0.01 & Pvs. $F(p=0.001)$ \\
\hline Comments on Medical & 3.07 & 0.84 & 3.56 & 0.80 & 2.95 & 0.84 & 3.05 & 0.68 & 98.25 & 2 & $<0.001$ & 0.07 & S vs. $F(p<0.001)$ \\
\hline School (4-image average) & & & & & & & & & & & & & S vs. $P(p<0.001)$ \\
\hline Parties or Dancing & 3.27 & 0.91 & 3.53 & 0.84 & 3.25 & 0.94 & 3.08 & 0.78 & 29.08 & 2 & $<0.001$ & 0.02 & S vs. $P(p<0.001)$ \\
\hline \multirow[t]{2}{*}{ (2-image average) } & & & & & & & & & & & & & S vs. $F(p<0.001)$ \\
\hline & & & & & & & & & & & & & P vs. $F(p=0.024)$ \\
\hline Opposite-Sex Pairs & 3.67 & 0.75 & 3.87 & 0.67 & 3.66 & 0.78 & 3.45 & 0.65 & 40.72 & 2 & $<0.001$ & 0.03 & S vs. $F(p<0.001)$ \\
\hline \multirow[t]{2}{*}{ (3-image average) } & & & & & & & & & & & & & S vs. $F(p<0.001)$ \\
\hline & & & & & & & & & & & & & P vs. $F(p<0.001)$ \\
\hline
\end{tabular}

* S vs. $\mathrm{P}=$ Students versus Public; S vs. F = Students versus Faculty; P vs. F = Public versus Faculty; NSD = No significant differences

* Given evidence of non-normality for some of our Likert-based measures, we used non-parametric Kruskall-Wallis $\mathrm{H}^{\prime}$ tests, which are adjusted for tie ranks, and follow the chi-squared distribution

$\uparrow$ Observed values of eta-squared $\left(=\mathrm{H}^{\prime} /[\mathrm{n}-1]\right)$ indicate small to moderate effect sizes (i.e. 0.01 range values suggest small effects; 0.06 range values are moderate; 0.14 are large)

\# Survey item: 'Please rate the appropriateness of what is CIRCLED IN RED in the above image'; 5 -point scale, reverse-coded: $1=$ Not at all appropriate to $5=$ Very appropriate

$\S$ Overall, findings suggest that faculty members identify the images as least appropriate, with students being the most permissive, and patients or others falling in between

If Survey item: 'Would you feel comfortable having the student who posted this as your doctor in the future?'; 5-point scale, reverse-coded: 1 = Absolutely would NOT feel comfortable to $5=$ Would be VERY comfortable

** Overall, findings suggest that faculty members and public (non-health care professionals) express less comfort with having the posting student as a future doctor, with students being more permissive

than students about the appropriateness of content posted on social media sites. There was a highly significant but somewhat weak global association between group membership and an overall appropriateness rating, calculated by averaging respondent assessment of all 29 images (Kruskal-Wallis $H^{\prime}=28.62$, d.f. $\left.=2, \mathrm{p}<0.001 ; \eta^{2}=0.02\right)$. Although
Dunn-Bonferroni-adjusted post hoc comparisons also showed a significant but fairly small difference between students $($ mean $=2.84)$ and the public $($ mean $=2.68)$ on this overall measure $(p=0.005)$, the difference between students and faculty members $($ mean $=2.53$ ) was also significant but larger in size. Note that for these measures, lower ratings 
Table 4 Enter-method multiple linear regression analysis: predictors of average appropriateness and comfort ratings (negative coefficients indicate lower or declining appropriateness or comfort ratings) $\dagger$

\begin{tabular}{|c|c|c|c|}
\hline & Model statistics & $\begin{array}{l}\text { Overall 'appropriateness' rating } \\
(29 \text {-image average) } \\
F=15.34, \text { d.f. }=10,1380, p<0.001 \\
R^{2}=0.100, \text { Cohen's } f^{2} \text { (effect size) }=0.11^{\Phi} \\
B \text { (beta) } p \text { value }\end{array}$ & $\begin{array}{l}\text { Overall 'comfort' with having the } \\
\text { student who posted the content } \\
\text { as a doctor ( } 29 \text {-image average) } \\
F=15.53 \text {, d.f. }=10,1380, p<0.001 \\
R^{2}=0.101, \text { Cohen's } f^{2} \text { (effect size) }=0.11^{\text {I }} \\
\text { B (beta) } p \text { value }\end{array}$ \\
\hline \multirow[t]{2}{*}{ Group } & Faculty member & $-0.152(-0.080) 0.003 *$ & $-0.035(-0.018) 0.505$ \\
\hline & Student & $-0.060(-0.034) 0.254$ & $0.149(0.082) 0.006 * *$ \\
\hline Gender & Female & $-0.248(-0.180)<0.001 * * *$ & $-0.252(-0.178)<0.001 * * *$ \\
\hline \multirow[t]{6}{*}{ Race/ethnicity } & $\begin{array}{l}\text { American Indian/ } \\
\text { Alaska Native }\end{array}$ & $-0.113(-0.009) 0.724$ & $-0.159(-0.012) 0.626$ \\
\hline & Asian & $-0.344(-0.039) 0.129$ & $-0.304(-0.034) 0.190$ \\
\hline & Black & $-0.082(-0.023) 0.362$ & $0.026(0.007) 0.776$ \\
\hline & $\begin{array}{l}\text { Hawaiian/Pacific } \\
\text { Islander }\end{array}$ & $-0.065(-0.031) 0.239$ & $-0.169(-0.080) 0.003 * *$ \\
\hline & Hispanic/Latino & $-0.330(-0.075) 0.003 * *$ & $-0.295(-0.066) 0.011^{*}$ \\
\hline & Multiracial & 0.393 (0.055) 0.033* & $0.194(0.026) 0.304$ \\
\hline Age & Age & $-0.013(-0.250)<0.001 * * *$ & $-0.012(0.215)<0.001 * * *$ \\
\hline \multicolumn{4}{|c|}{$\begin{array}{l}* p<0.05, * * p<0.01, * * * p<0.001 \\
\dagger \text { In this enter-method linear regression, all variables were entered as a single block (i.e. simultaneous forced entry). For the variable } \\
\text { group, the excluded/comparison category = public; for gender, the excluded/comparison category = male; for race/ethnicity, the } \\
\text { excluded/comparison category = Caucasian } \\
\$ \text { Survey item: 'Please rate the appropriateness of what is CIRCLED IN RED in the above image' (5-point scale, reverse-coded: } 1=\mathrm{NOT} \text { at } \\
\text { all appropriate to } 5=V \text { VRY appropriate). Statistically significant findings indicate that faculty members, females and Hispanic/Latino } \\
\text { respondents identified a greater degree of 'inappropriateness', whereas multiracial respondents identified less. For age, older respondents } \\
\text { identified less appropriateness } \\
\S \text { Survey item: 'Would you feel comfortable having the student who posted this as your doctor in the future?' (5-point scale, reverse- } \\
\text { coded: } 1=\text { absolutely would NOT feel comfortable to } 5=\text { would be VERY comfortable). Statistically significant findings indicate that } \\
\text { female, Hawaiian/Pacific Islander and Hispanic/Latino respondents indicated more discomfort with having the posting student as a doctor, } \\
\text { whereas as a group, students indicated less overall discomfort. For age, older respondents expressed more discomfort } \\
\text { I The observed Cohen's } \mathrm{f}^{2} \text { values suggest small to moderate effect sizes, given the convention that } 0.02 \text { range values indicate small, } 0.15 \\
\text { range values indicate moderate and } 0.35 \text { range values indicate large effects. Cohen's } \mathrm{f}^{2}=\left(\mathrm{R}^{2} /\left[1-\mathrm{R}^{2}\right] \text { ) }\right.\end{array}$} \\
\hline
\end{tabular}

indicated respondents found images to be less appropriate.

There was also a highly significant but weak to moderate global association between group membership and average comfort rating, based on ratings of comfort with having the students who posted each of the 29 images as their doctor in the future (Kruskal-Wallis $H^{\prime}=51.44$, d.f. $=2, \mathrm{p}<0.001$; $\left.\eta^{2}=0.04\right)$. Post hoc comparisons identified significant differences between students (mean $=3.03$ ) and the public $($ mean $=2.70)(p<0.001)$, as well as a significant difference between students and faculty members $($ mean $=2.67)(p<0.001)$. Again, lower averages indicated respondents were less comfort- able with having the student(s) who posted the image as their doctor in the future.

In addition to results for the 29-image averages, Table 3 also presents the image topics categorised and sorted from least appropriate to most appropriate according to overall (total) respondent ratings. In terms of appropriateness, of the 11 screenshot topics, faculty members indicated statistically significant lower appropriateness compared with medical students in eight categories (post hoc comparison $\mathrm{p}$ values were all $<0.001)$. The public's appropriateness ratings were significantly lower than those of medical students in six topic areas (with $\mathrm{p}$ values ranging from $<0.001$ to 0.018 ). There were similar 
findings in terms of comfort with having the posting students as a future doctor. Faculty member comfort ratings were significantly lower than medical student ratings in nine categories ( $p$ values from $<0.001$ to 0.022 ) and the public's ratings were significantly lower than those of medical students in eight categories (all $\mathrm{p}$ values in the $<0.001$ range).

Overall, respondents rated images that contained comments on patients as the most inappropriate, followed by an image that included marijuana. They rated images that showed opposite-sex pairs as least inappropriate. In terms of comfort with having the student who posted the image as their doctor, respondents rated the same two topics (comments on patients and marijuana) as causing the most discomfort and the same (opposite-sex pairs) causing the least discomfort (Table 3). Differences in appropriateness ratings among the groups were statistically significant $(p<0.001)$ in every category except comments on patients and clinical picture-domestic. Differences in comfort level with the posting student becoming their doctor were significantly different $(p<0.011)$ in every category except clinical picture-domestic.

Table 4 summarises the results from two multiple linear least-squares regression analyses. Independent variables were added using the simultaneous forcedentry approach (i.e. enter method, with all variables added at once). The first regression uses the 29image average from the 'appropriateness' items as the dependent variable $(F=15.34$, d.f. $=10,1380$, $\mathrm{p}<0.001 ;$ Cohen's $f^{2}=0.11$, suggesting a moderate effect size), whereas the second uses the 29-image average from the 'comfort with having this student as a doctor' items as the dependent variable $(F=15.53$, d.f. $=10,1380, \mathrm{p}<0.001$; Cohen's $f^{2}=0.11$, suggesting a moderate effect size). Statistically significant differences for group effects persisted after controlling for age, gender and raceethnicity. In keeping with trends observed in the Kruskal-Wallis $H^{\prime}$ analyses, faculty members corresponded to lower, more conservative ratings (e.g. $B=-0.150, \mathrm{p}=0.003$ for the average 'appropriateness' rating) and students corresponded to higher, more liberal ratings (e.g. $B=0.149, \mathrm{p}=0.006$ for 'comfort'). We also noted persistent effects for respondent gender, with females evaluating the images as less appropriate $(B=-0.248, p<0.001)$ and expressing greater discomfort with those who posted them $(B=-0.252, \mathrm{p}<0.001)$, after controlling for other variables in the model. Those in the multiracial category were significantly more liberal in evaluating appropriateness $(B=0.393, \mathrm{p}=0.033)$ and those in the Hispanic/Latino category were more conservative $(B=-0.333, \mathrm{p}=0.003)$. Similarly, those in the Hawaiian/Pacific Islander and Hispanic/Latino categories were significantly more conservative in evaluating comfort with students who posted the content as their future doctors $(B=-0.169, \mathrm{p}=0.003$ and $B=-0.295, \mathrm{p}=0.011$, respectively). Finally, age persisted as a significant effect in both models, with $B=-0.013(\mathrm{p}<0.001)$ in the 'appropriateness' model and $B=-0.012$ $(\mathrm{p}<0.001)$ in the 'comfort' model. These findings for age indicate that older respondents are more conservative, providing lower appropriateness and comfort ratings.

\section{DISCUSSION}

The widespread use of social media is fairly recent and much more prevalent among younger individuals, ${ }^{11}$ but the number of Facebook ${ }^{\circledR}$ users in all age groups has increased over the past 5 years, including those in the 'over 65 years of age' group. ${ }^{22}$ Use of Facebook ${ }^{\circledR}$ by members of all groups studied was consistent with usage patterns reported by reviews of social media usage among different demographic groups, in which a higher percentage of younger adults use Facebook ${ }^{\circledR}$ to create and maintain connections with others. ${ }^{21}$ We made some novel observations regarding different viewpoints among the groups studied regarding the appropriateness of posting particular content as a future doctor. In general, faculty members and the public were more conservative than students about the appropriateness of content posted on social media sites. Overall, our findings extend previous research on the content of medical trainees' Facebook ${ }^{\circledR}$ profiles and postings and expand our understanding about disparate perceptions regarding social media professionalism among key stakeholders in academic medicine.

In our study we found significant differences in perceptions of appropriateness as rated by three groups: medical school faculty members, medical students and the public. Although our study included simulated screenshots (Fig. S1), the content mirrored actual content found posted by medical students, such as excess alcohol use, overt sexuality, profanity and patient privacy violations, as also found by Thompson and colleagues. ${ }^{14}$ Of note, faculty members indicated statistically significant lower appropriateness and comfort levels compared with medical students in slightly more categories 
than the public's appropriateness ratings, suggesting that medical school faculty members expect the highest degree of professionalism among students than the general public (based on our sample). All respondents rated images that clearly violated current professional standards or laws, with comments on patients as the most inappropriate and causing the most discomfort, followed by an image with marijuana. Not surprisingly, given our societal norms, images depicting opposite-sex relationships (i.e. kissing) were viewed as least inappropriate and caused the least discomfort among all three groups. The finding that differences in both appropriateness and comfort level ratings were not statistically significant in one category, clinical picture-domestic, suggests that there is broad awareness among members of all groups regarding basic patient privacy issues.

In our survey we explored whether respondents felt content very similar to that posted by actual medical students was appropriate versus inappropriate. We felt that the more colloquial term 'inappropriate' conveyed equivalent social, behavioural and ethical connotations as denoted by the more disciplinebased term 'unprofessional'. Given this, we believe that our results provide an accurate reflection of perceptions of online professionalism among these stakeholder groups. In general, we found that medical students differed significantly from doctors and the public in terms of the types of social media posting they find appropriate versus inappropriate, and in terms of the consequences of such postings when viewed by individuals who are not medical students.

Our findings suggest that medical students are more likely to post comments, images and photographs that medical school faculty members and the public would consider inappropriate or unprofessional. Statistical differences were found among the three groups' ratings of the screenshots and persisted as a significant factor, even when we controlled for age.

Of additional interest is that certain images or comments revealed personal information about social network users. The basic Facebook ${ }^{\circledR}$ profile allows users to identify their sexual orientation publicly, as well as their political and religious ideologies. This information, which would probably not be revealed in a traditional patient encounter, could cause a patient to withhold information, to form preconceived biases or simply to feel uncomfortable with that individual as their doctor. We found this in our study - a higher percentage of the public group found images depicting same-sex relationships inap- propriate and they were less comfortable having the student posting the image as their future doctor than were members of the student group. The fact that same-sex relationships were viewed more negatively among faculty members and public groups when compared with the medical student group probably reflects cultural norms, where there is growing acceptance of same-sex relationships, especially among certain demographic groups in our society.

A major issue remains in how to help medical students understand that what they think is appropriate to post on a social media site is significantly different from what faculty members or the public, their future patients, might think, and that postings thought by others to be inappropriate could have professional or reputational consequences. We believe that our findings provide useful insights to help support discussions and awareness about online professionalism for medical students. We hope our findings will be useful for students in considering the perspectives of patients and faculty members more carefully when making decisions about content they are thinking about posting on their social media sites.

\section{Limitations}

It is important to consider our study in the context of its scope and limitations. Although we believe the respondents to our survey are representative of students, faculty members and the public from other academic medical centres in the country, the study was conducted at a single large Midwestern institution. Another limitation is that our screenshots of Facebook ${ }^{\circledR}$ profiles were created by several of the authors to depict specific content that is found on medical student sites, so they were not actual Facebook ${ }^{\circledR}$ profiles of current medical students.

Although unlikely, this may have influenced our results. Although we chose certain screenshots to represent specific types of behaviour (nudity, substance use, etc.), other variables presented in the images (i.e. gender, ethnicity, age, clothing, etc.) that were unaccounted for in our study may have influenced responses. Furthermore, the 29 screenshots were limited in scope in that we could have included more images of all of the categories to gain a more comprehensive understanding of the differences among the categories. For example, multiple images of opposite-sex and same-sex relationships, taking into account age, gender, race, clothing, setting, etc., and additional probing questions about what impacted respondents' perceptions about appropriateness would be needed to 
understand more clearly what variables impact perceptions beyond depicted sexual orientation. In addition, our study was based on the assumption that all profiles and, hence, screenshots were readily viewable and not blocked by privacy settings. However, as stated above, $89.8 \%$ of respondents use some form of privacy settings. A better understanding of how our respondents use these settings and how confident they are that these settings block unwanted individuals from viewing their profiles would have helped in interpreting out results. Lastly, an online survey made it difficult to measure and to understand intricate or nuanced differences regarding topics such as the appropriateness of certain screenshots.

\section{Summary and future directions}

We believe our findings have important implications for development of guidelines created to help medical students learn to reflect on content they intend to post before they post it. Although medical education has traditionally relied significantly on role modelling as a primary tool for learning about professionalism, given what appear to be age differences in how such sites are used and how content is viewed, role modelling about social networking might not be realistic or effective. However, discussion and data presentation about differences in generation and perception among groups with regard to sites like Facebook ${ }^{\circledR}$ might help to bring the issue to the forefront and provide students with evidence to reflect on in the context of what they choose to post. For example, sharing the outcomes of this study might bring awareness to both medical students and faculty members and allow for more collaboration on creating guidelines in the future. Helping students to understand professionalism in terms of their online identities might be more successful if framed in the context of their professional identity formation as medical trainees and ultimately doctors. Even entering medical students have a vision of themselves as doctorsin-training. Understanding professionalism in medicine and how that is portrayed through social media might be an excellent foundation for helping students to move from one stage of identify formation to the next.

Further research on this topic is vital to continuing this important conversation. We believe that our results support the need for further exploration of the implications of students' social media postings. In addition, studies that evaluate the approaches and effect of education regarding privacy settings and sharing of information with patients as opposed to attempting to regulate the content of information being posted by the medical students can provide valuable information to patients, students and faculty members.

Contributors: each of the authors contributed substantially to the conception and design of the study and to the acquisition of data. JF conducted the data analysis in consultation with the authors. AJ, EP and JW drafted the article. All authors revised it for critical content. JP (with JF) contributed to conceptualisation of the methodology and analysis of the data. All authors gave final approval of the version of the manuscript submitted.

Acknowledgements: the authors wish to acknowledge and thank Sujal Parikh for his assistance with this research study.

Funding: none.

Conflicts of interest: none.

Ethical approval: this study was granted exemption from informed consent by the institutional review board of the University of Michigan Health System.

\section{REFERENCES}

1 Experian Marketing Services. Hitwise. http://www. hitwise.com/us/datacenter/main/dashboard-10133. html. [Accessed 10 December 2011.]

2 Facebook. Newsroom: Key Facts. 2013. http:// newsroom.fb.com/content/default.aspx? NewsAreaId=22, 2012. [Accessed 10 December 2011.]

3 Bosslet GT, Torke AM, Hickman SE, Terry CL, Helft PR. The patient-doctor relationship and online social networks: results of a national survey. J Gen Intern Med 2011;26:1168-74.

4 Modahl M, Tompsett L, Moorhead T. Doctors, patient \& social media. Quantia MD 2011;September 2011. http: / / www.google.com/ url?sa=t\&rct=j\&q=\&esrc=s \&source=web\&cd=1\&ved=0CC0QFjAA\&url=http $\% 3 \mathrm{~A} \%$ 2F\%2Fwww.quantiamd.com\%2Fq-qcp\%2FDoctors PatientSocialMedia.pdf\&ei=kqHmUZOEArHj4API4I CgDQ\&usg=AFQjCNF7tP42bEZ5b18Tg4f1eA08itahW Q\&sig2=dBHwvfS0m0Ordw1663Rt9g\&bvm=bv.49405 654,d.dmg. [Accessed 17 July 2013.]

5 Kind T, Genrich G, Sodhi A, Chretien KC. Social media policies at US medical schools. Med Educ Online 2010;15.

6 Greysen SR, Kind T, Chretien KC. Online professionalism and the mirror of social media. J Gen Intern Med 2010;25:1227-9.

7 Mostaghimi A, Crotty BH. Professionalism in the digital age. Ann Intern Med 2011;154:560-2.

8 Snyder L, American College of Physicians Ethics, Professionalism, and Human Rights Committee. American College of Physicians Ethics Manual: sixth edition. Ann Intern Med 2012;156(1 Pt 2):73-104. 
9 Australian Medical Council. Good medical practice: a code of conduct for doctors in Australia. 2013. http://www.amc.org.au/index.php/about/ good-medical-practice. [Accessed January 2013.]

10 Mayer MA, Leis A, Mayer A, Rodriguez-Gonzalez A. How medical doctors and students should use social media: a review to propose some practical recommendations. Stud Health Technol Inform 2012;180:853-7.

11 Chretien KC, Farnan JM, Greysen SR, Kind T. To friend or not to friend? Social networking and faculty perceptions of online professionalism. Acad Med 2011;86:1545-50.

12 Chretien KC, Goldman EF, Beckman L, Kind T. It's your own risk: medical students' perspectives on online professionalism. Acad Med 2010:85 (Suppl 10):S68-71.

13 Finn G, Garner J, Sawdon M. 'You're judged all the time!' Students' views on professionalism: a multicentre study. Med Educ 2010;44:814-25.

14 Thompson LA, Dawson K, Ferdig R et al. The intersection of online social networking with medical professionalism. J Gen Intern Med 2008;23:954-7.

15 Chretien KC, Greysen SR, Chretien JP, Kind T. Online posting of unprofessional content by medical students. JAMA 2009;302:1309-15.

16 Garner J, O'Sullivan H. Facebook and the professional behaviours of undergraduate medical students. Clin Teach 2010;7:112-5.

17 Jarvis-Selinger S, Pratt DD, Regehr G. Competency is not enough: integrating identity formation into the medical education discourse. Acad Med 2012;87: 1185-90.

18 Kaczmarczyk JM, Chuang A, Dugoff L et al. eProfessionalism: a new frontier in medical education. Teach Learn Med 2013;25:165-70.

19 Sandars J, Schroter S. Web 2.0 technologies for undergraduate and postgraduate medical education: an online survey. Postgrad Med J 2007;83:759-62.

20 Sandars J, Homer M, Pell G, Croker T. Web 2.0 and social software: the medical student way of e-learning. Med Teach 2008;30:308-12.

21 Duggan M, Brenner J. The demographics of social media users - 2012. Pew Internet and American Life Project, 14 February 2013.

\section{SUPPORTING INFORMATION}

Additional Supporting Information may be found in the online version of this article:

Figure S1. Examples of survey items and specific questions. The images shown are examples of fabricated mock screenshots used in the survey.

Received 26 June 2012; editorial comments to author 4 September 2012, 5 March 2013; accepted for publication 4 June 2013 